\title{
Speed breeding is a powerful tool to accelerate crop research and breeding
}

\begin{abstract}
The growing human population and a changing environment have raised significant concern for global food security, with the current improvement rate of several important crops inadequate to meet future demand 1 . This slow improvement rate is attributed partly to the long generation times of crop plants. Here, we present a method called 'speed breeding', which greatly shortens generation time and accelerates breeding and research programmes. Speed breeding can be used to achieve up to 6 generations per year for spring wheat (Triticum aestivum), durum wheat (T. durum), barley (Hordeum vulgare), chickpea (Cicer arietinum) and pea (Pisum sativum), and 4 generations for canola (Brassica napus), instead of 2-3 under normal glasshouse conditions. We demonstrate that speed breeding in fully enclosed, controlled-environment growth chambers can accelerate plant development for research purposes, including phenotyping of adult plant traits, mutant studies and transformation. The use of supplemental lighting in a glasshouse environment allows rapid generation cycling through single seed descent (SSD) and potential for adaptation to larger-scale crop improvement programs. Cost saving through light-emitting diode (LED) supplemental lighting is also outlined. We envisage great potential for integrating speed breeding with other modern crop breeding technologies, including high-throughput genotyping, genome editing and genomic selection, accelerating the rate of crop improvement.
\end{abstract}

Keyword: Agriculture; Biological techniques; Plant breeding; Plant molecular biology; Plant sciences 